when warmed gently. The residue from the ammonia filtrate presented the same appearance but was not soluble in alcohol and ether, but readily taken up by ammonia.

Both filtrates on evaporation threw out the dissolved matter in brownish flocks. We hope to investigate this point more thoroughly and also the action of ammonia on the total carbon of pig iron as obtained by use of the double chlorides. Such work may aid in obtaining a better knowledge of the forms of combination of carbon with iron and the forms in which it is separated under different conditions.

Attention will also be paid to the character of spots on the crucible, but these would not be common to all pig iron and might exist to a greater extent in some than in others. I may note in conclusion that all filtrations were performed by aid of the pump, and that more closely concordant results might have been obtained had the filtrations been performed upon asbestos. It would also be interesting to determine whether the silicon of the metal under this treatment is rolatilized or largely in solution.

I.ABORATORY OF STEWART IRON CO., L'T'Y

SHARON, PA., DECEMBER, IBÜ.

\title{
THE DETECTION OF STRYCHNINE IN AN EXHUMED HUMAN BODY.'
}

I3Y W. A. Nosfs.

DROFESSOR T. G. Wormley in his book on the MicroChemistry of Poisons states that strychnine has never been found in an exhumed human body after a longer period of burial than forty-three days. His authority is the Ann. d'Hyg., April, 7881,359 . I have not been able to find in any chemical journal a contradiction of this statement, though there is a statement in the old edition of Watt's Chemical Dictionary which seems to be inconsistent with it. Under these circumstances the following account of a recent toxical case may be of some interest.

On April 26th of this year Drs. W. L. Athon and O. Mitchell, of Marshall, I11., brought to me for examination the stomach, liver and a portion of the intestines of a child who died on June

1 Read at the Baltimore Meeting, December 28,1893 . 
23rd, 1892, and was buried the following day. The body was exhumed on April 25th, 1893, after a burial of 308 days. The material submitted for examination weighed 505 grams. About fifty grams were examined for arsenic and other mineral poisons with negative results. A bout 350 grams of the remainder were extracted with dilute sulphuric acid and alcohol by the usual methods. The aqueous residue which remained on concentration of the alcoholic solution contained a considerable amount of oily matter which was separated by filtration and washed as thoroughly as possible. This residue (A) is usually considered to be nearly free from alkaloids but it was further examined as described below.

The filtrate, after concentration, treatment again with alcohol, etc., was rendered alkaline with caustic soda and extracted with chloroform. The chloroform left, on evaporation, a considerable amount of oily bases which were partly volatile, as was shown by their fumes with hydrochloric acid. To separate the strychnine from this residue it was heated on a water-bath with a little concentrated sulphuric acid for two hours. After neutralizing the acid the solution was extracted with chloroform. The residue obtained, was treated again in the same manner, and that residue, again. Stoeh ${ }^{1}$ has shown that when pure strychnine is heated to $100^{\circ}$ with pure concentrated sulphuric acid, for some time, it is completely converted into the mono-sulphonic acid. Apparently in the presence of much foreign matter and with a small amount of acid in an open watch glass a considerable portion, at least, of the strychnine escapes this action of the acid. In any case, a portion of the last residue referred to above gave with potassium pyrochromate and sulphuric acid the violet color, changing after a short time to an onion red, which is characteristic of strychnine. While the use of concentrated sulphuric acid is open to objection and should never be resorted to when it is possible to avoid it, I do not know of any other method which would be successful in the case of such residues and such minute amounts of strychnine as were obtained in this case.

The oily residue (A) was treated with some purified ligroin, 1 Ber. d.chem. Ges., 18, 3429. 
and very dilute sulphuric acid and the mixture was filtered with the aid of a pump in such a manner that both acid and ligroin were drawn through the filter. The acid solution and the ligroin were then separated and the strychnine was recovered from the solution and purified essentially as before. A considerably larger amount was obtained from this portion than from the first. The strychnine obtained was identified by the characteristic reaction with potassium pyrochromate and sulphuric acid, by the intensely bitter taste, by the crystalline form and clouble refraction as seen with the microscope, by the crystals obtained from the chloride with potassium chromate, and by the effect of about is of a milligram upon a frog weighing about two grams. The frog died from the effects of the poison, the symptoms of tetanus being fully developed within ten minutes after the close was administered. The crystals of the chromate were chiefly of the branching forms; there was a little evidence of octahedra but they were not positively identified. The branching forms, however. developed the characteristic violet color when touched with concentrated sulphuric acid.

The total amount of strychnine recovered was estimated to be about two milligrams.

In the trial the chemical evidence was not contested. The defendant was, however, acquitted on other grounds.

Rosi: DULYTECHNK INSTTUT:

DECEMUR 23, 2802

\section{NOTES ON DETERMINATION OF NICKEL IN STEEL.'}

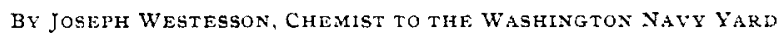

T

$\mathrm{HE}$ desirability of being able to determine nickel in steel

by means of a shorter process than the one, now mostly in use, gave me cause some time ago, when a number of sanples from nickel-steel plates were submitted for analysis to the ordnance laboratory, and when the tedioumess of the said process became particularly apparent, to make some investigations in order to find a more direct method, and I shall beg permission to mention in a few words, what my trials led to.

In the process, above mentioned, the nickel is precipitated

1 Read bytitle at the Baltimore meeting, December 23,1893 . 